\title{
Equilibrium Piezoelectric Potential Distribution in a Deformed ZnO Nanowire
}

\author{
Giulia Mantini ${ }^{1,2}$, Yifan Gao ${ }^{1}$, A. D’Amico ${ }^{2}$, C. Falconi ${ }^{2}$, and Zhong Lin Wang ${ }^{1}(\bowtie)$ \\ ${ }^{1}$ School of Materials Science and Engineering, Georgia Institute of Technology, Atlanta, Georgia 30332-0245, USA \\ ${ }^{2}$ Department of Electronic Engineering, University of Tor Vergata, Via del Politecnico 1, 00133 Roma, Italy \\ Received: 20 April 2009 / Revised: 21 May 2009 / Accepted: 21 May 2009 \\ (C) Tsinghua University Press and Springer-Verlag 2009. This article is published with open access at Springerlink.com
}

\begin{abstract}
The equilibrium piezoelectric potential distribution in a deformed $\mathrm{ZnO}$ semiconductive nanowire has been systematically investigated in order to reveal its dependence on the donor concentration, applied force, and geometric parameters. In particular, the donor concentration markedly affects the magnitude and distribution of the electric potential. At a donor concentration of $N_{\mathrm{D}}>10^{18} \mathrm{~cm}^{-3}$, the piezopotential is almost entirely screened. Among the other parameters, a variation in the length of the nanowire does not significantly affect the potential distribution.
\end{abstract}

\section{KEYWORDS}

Nanogenerator, $\mathrm{ZnO}$, nanowire

Energy harvesting is gaining more and more importance in the development of self-powered devices for different applications, such as implanted biomedical devices, environmental monitoring, wireless sensor networks, and personal electronics. Piezoelectricity is one of the most convenient methods for harvesting energy from the environment. Mechanical energy can be harvested from a variety of sources such as body movement and muscle stretching, acoustic waves, and blood flow, and can be converted into electricity by the piezoelectric effect.

Piezoelectric nanogenerators using nanowires (NWs) have been recently reported for converting mechanical energy into electricity on the nanoscale $[1,2]$. NWs made of wurtzite structured materials, in particular of $\mathrm{ZnO}$, may take advantage of both the semiconducting and piezoelectric properties of these materials to scavenge energy from the environment, The principles of a nanogenerator have already been established [3-6]. A piezoelectric potential is created in the NW as a result of elastic deformations produced by an external force; this potential can drive the flow of charge carriers through an external load. A Schottky barrier formed at the junction of the NW with the electrode controls the flow direction of the charge carriers [3]. This principle has been used in different systems, such as a single nanowire bent by the tip of an atomic force microscope (AFM) [3] and an array of aligned NWs with a zigzag top electrode and with ultrasonic excitation [4]. Furthermore, based on the coupled piezoelectric and semiconducting properties of NWs, a new field of nanopiezotronics has been introduced [7], which utilizes the piezoelectric potential to modulate the

Address correspondence to zlwang@gatech.edu 
carrier transport process in the NW itself for building various electronic devices and components, such as transistors and diode.

The theoretical basis of the nanogenerator and nanopiezotronics arises from the voltage drop created across the cross section of the NW when it is laterally deflected. The surface of the compressed side of the NW will exhibit a negative potential, while the surface of the stretched side will present a positive potential. The piezoelectric potential is created by the polarization of anions and cations inside the NW and will remain as long as the NW is maintained in a deformed configuration, because the polarization charges cannot freely move. This continuum model for the electrostatic potential in a bent piezoelectric NW has been presented in Ref. [8], and also applied in Ref. [9]. This first model and associated calculations were based on the Lippman theory [10], which can only be used when the donor concentration is extremely low, so that the conductivity of the material can be neglected.

However, $\mathrm{ZnO}$ NWs epitaxially grown along the $c$ axis on a single crystal substrate are typically n-type due to unavoidable point defects [11-13]. Thus, in order to model a semiconductor material with a significant amount of free electrons, which are indeed able to distribute all over the material, the Lippman theory cannot be directly applied. In Ref. [14], a new model has been presented in order to investigate the statistics of electrons/holes and obtain a more realistic picture of the operation mechanism of piezoelectric nanogenerators. A macroscopic-statistical model of piezoelectricity in a laterally bent semiconductive nanowire with moderate conductivity in a normal doping range was presented. In particular, the electric potential when thermodynamic equilibrium among free charge carriers is achieved has been calculated. In Ref. [14] the calculations were conducted exclusively for n-type $\mathrm{ZnO}$ nanowires, but as the methodology is directly applicable to any similar piezoelectric and semiconductive material with the same design, the calculation for a p-type $\mathrm{ZnO}$ nanowire has also been carried out [15].

The main objective of this paper is to systematically investigate the influence of different parameters on the equilibrium piezoelectric potential distribution in a deformed $\mathrm{ZnO}$ semiconductive NW, according to the methods and results described in Ref. [14]. In particular, we will calculate the electric potential distribution when the thermodynamic equilibrium among free charge carriers is achieved for NWs with different doping concentrations, different applied forces, and different geometric configurations.

The theoretical framework of these calculations, already presented in Ref. [14], will be briefly reviewed here. When considering a semiconductive piezoelectric material, the free electrons/hole carriers redistribute in accordance with the electric field created by the polarization. So in order to investigate the behavior of the deformed nanowire we will need to take into account both the piezoelectric constitutive equation and the redistribution of electrons under thermodynamic equilibrium, given by the FermiDirac statistics. The piezoelectric fully coupled constitutive equation is given by

$$
\left\{\begin{array}{c}
\sigma_{\mathrm{p}}=c_{\mathrm{pq}} \varepsilon_{\mathrm{q}}-e_{\mathrm{kp}} E_{\mathrm{k}} \\
D_{\mathrm{i}}=e_{\mathrm{iq}} \varepsilon_{\mathrm{q}}+\kappa_{\mathrm{ik}} E_{\mathrm{k}}
\end{array}\right.
$$

where $\sigma$ is the stress tensor, $\varepsilon$ is the strain tensor, $E$ is the electric field, $D$ is the electric displacement, $\kappa_{\mathrm{ik}}$ is the dielectric constant, $e_{\mathrm{iq}}$ is the piezoelectric constant, and $c_{\mathrm{pq}}$ is the mechanical stiffness tensor. It has already been shown in Ref. [8] that the fully coupled constitutive equation can be replaced, without loss of generality, by the mechanical equilibrium and the direct piezoelectric effect, thus neglecting only the term $-e_{\mathrm{kp}} E_{\mathrm{k}}$ relative to the indirect piezoelectric effect. The mathematical description of the Fermi-Dirac statistics is given by

$$
\left\{\begin{array}{l}
n=N_{\mathrm{c}} F_{1 / 2}\left(-\frac{E_{\mathrm{c}}(x)-E_{\mathrm{F}}}{k T}\right) \\
N_{\mathrm{c}}=2\left(\frac{2 \pi m_{\mathrm{e}} k T}{h^{2}}\right)^{3 / 2}
\end{array}\right.
$$

where $E_{\mathrm{c}}$ is the conduction band edge, $E_{\mathrm{F}}$ is the Fermi level, and $N_{c}$ is the effective state density of conduction band, which is determined by the effective mass of conduction band electron $m_{\mathrm{e}}$ and the temperature $T$. Finally, the activation process of the donors must be taken into account:

$$
N_{\mathrm{D}}^{+}=N_{\mathrm{D}} \frac{1}{1+2 \exp \left(\frac{E_{\mathrm{F}}-E_{\mathrm{D}}}{k T}\right)}
$$


where $E_{\mathrm{D}}$ is the position-dependent donor energy level and $N_{\mathrm{D}}$ is the concentration of the donors. Equations (1)-(3) together with Gauss's Law allow the calculation of the equilibrium piezoelectric potential in a deformed nanowire, considering its charge carrier density distribution. Both the nanowire and the substrate on which the nanowire is grown are modeled as made of $\mathrm{ZnO}$. The dimensions of the substrate are much larger than that of the nanowire, so that the substrate acts as a vast reservoir that pins the Fermi level.

The finite element method (FEM) was used to solve the nonlinear partial differential equations described above (and explained in more detail in Ref. [14]). A ZnO nanowire epitaxially grown along the $c$ axis is laterally bent by a force $f_{y}$ exerted at the top. An axially symmetric model is chosen in order to simplify the calculations. This means that the equations are solved for the half-space $x>0$ and the solutions are eventually derived using the mirror symmetry of the $x=0$ plane.

The material constants for $\mathrm{ZnO}$ used in these calculations are chosen as in Ref. [14]: Young's modulus $E=129 \mathrm{GPa}$; Poisson ratio $v=0.349$; relative dielectric constants $\kappa_{\infty}=7.77, \kappa_{\|}=8.91$; piezoelectric constants $e_{31}=-0.51 \mathrm{C} / \mathrm{m}^{2}, e_{33}=1.22 \mathrm{C} / \mathrm{m}^{2}, e_{15}=-0.45$ $C / m^{2}$; effective mass $m^{*}=0.628 m_{0}$; other physical parameters such as the activation energy of donors are adopted from Ref. [14]. As for the geometry, the nanowire is modeled as a cylinder. The force is uniformly applied on the top surface of the nanowire perpendicular to its axis.

Figure 1 shows the influence of the donor concentration on both the equilibrium piezoelectric potential and the local electron density. The colorplot of the piezoelectric potential in Figs. 1(a), 1(b),

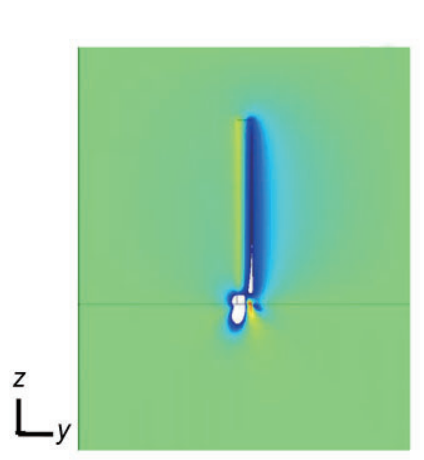

(a)

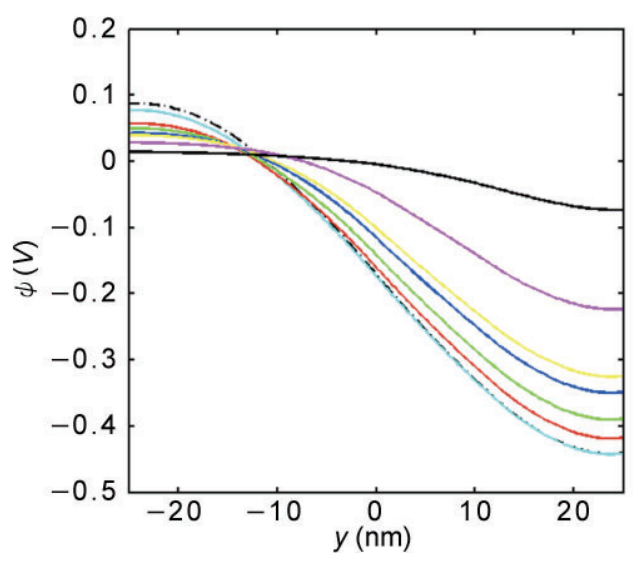

(d)

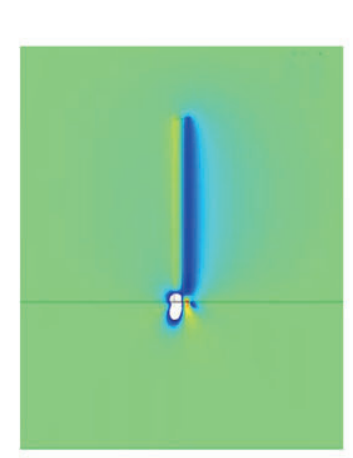

(b)

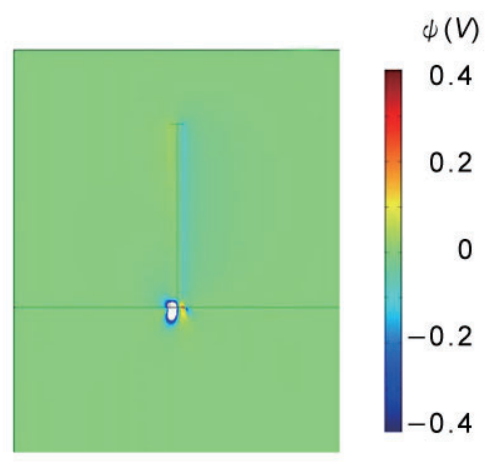

(c)

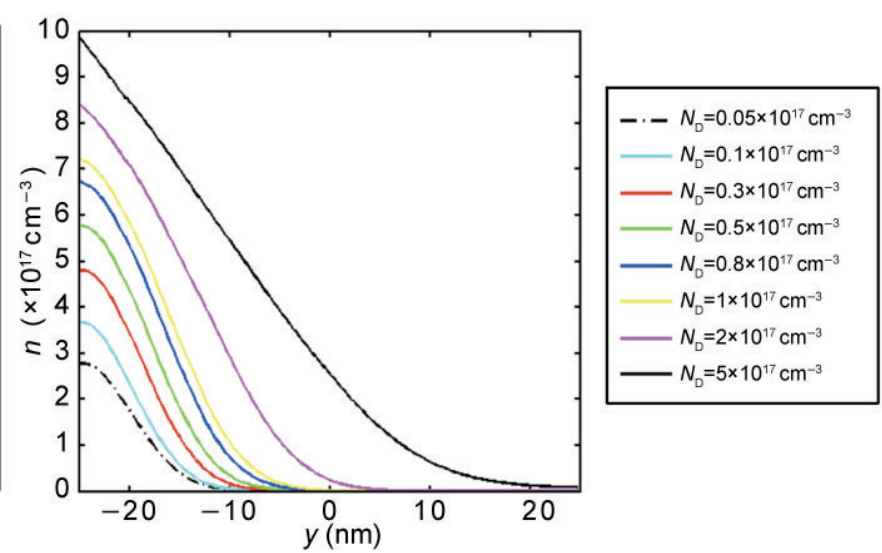

(e)

Figure 1 Color-plot of the calculated piezoelectric potential at a cross section of $x=0$, for donor concentrations $N_{D}=0.5 \times 10^{17} \mathrm{~cm}^{-3}(a)$, $N_{D}=1 \times 10^{17} \mathrm{~cm}^{-3}(\mathrm{~b})$ and $N_{D}=5 \times 10^{17} \mathrm{~cm}^{-3}$ (c). The dimensions of the nanowire are $L=600 \mathrm{~nm}$ and $\mathrm{a}=25 \mathrm{~nm}$; the external force is $f_{y}=80 \mathrm{nN}$. (d) Piezoelectric potential and (e) local electron density for different donor concentrations $0.05 \times 10^{17} \mathrm{~cm}^{-3}<N_{D}<5 \times 10^{17} \mathrm{~cm}^{-3}$, for $T=300 \mathrm{~K}$. The line plot is along the diameter of the nanowire, at $z=400 \mathrm{~nm}$ 
and 1 (c) represents the results of low $\left(0.5 \times 10^{17} \mathrm{~cm}^{-3}\right)$, medium $\left(1 \times 10^{17} \mathrm{~cm}^{-3}\right)$, and high $\left(5 \times 10^{17} \mathrm{~cm}^{-3}\right)$ donor concentrations $N_{\mathrm{D}}$, respectively. In Figs. 1(d) and 1(e) we plot the electric potential $\varphi$ and the free electron concentration $n$ for different donor concentrations between $0.05 \times 10^{17} \mathrm{~cm}^{-3}$ and $5 \times 10^{17} \mathrm{~cm}^{-3}$, at $T=300$ $\mathrm{K}$. The electric potential $\varphi$ in the stretched side, showing a positive potential, is less sensitive than the compressed negative side to the increase of donor concentration $N_{\mathrm{D}}$. The electric potential $\varphi$ is almost completely screened for $N_{\mathrm{D}}=5 \times 10^{17} \mathrm{~cm}^{-3}$. The reason for the screening of the potential in the compressed side of the nanowire is that free electrons will be depleted in this region, while they will accumulate at the stretched side. Moreover, the decrease of the positive potential is due to the inflow of free electrons from the substrate reservoir, where free charges are abundant. This increase in free electron concentration is clearly evident in Fig. 1(e).

Figure 2 shows the influence of the applied force on the equilibrium potential distribution and local electron density. All of the other parameters are kept constant: nanowire length $600 \mathrm{~nm}$, radius $25 \mathrm{~nm}$, and donor concentration $N_{\mathrm{D}}=1 \times 10^{17} \mathrm{~cm}^{-3}$. The force has been chosen to vary in the range 40 $\mathrm{nN}<F<140 \mathrm{nN}$. In the calculations, the force was

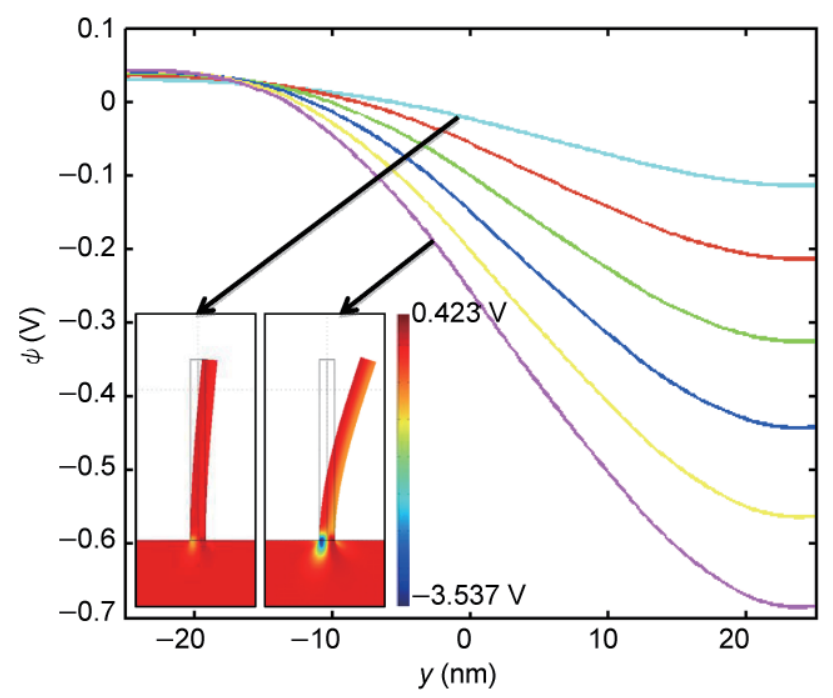

(a) applied to the top surface of the nanowire in order to avoid punctual deformations. When increasing the applied force, the electric potential (in Fig. 2(a)) of the compressed side increases, reaching a value of about $0.7 \mathrm{~V}$ for the maximum applied force. The free electron concentration (Fig. 2(b)) also increases in the stretched side when increasing the force, because of the increase in the polarization charges due to the highest strains. Figure 2(a) also shows the color-plots of the electric potential and the scaled deformations for lower and higher applied forces. Figure 2(b) shows the color-plot for the free electron concentration, at the cross-section at $z=400 \mathrm{~nm}$. This last figure is obtained by means of the mirror symmetry of the $x=0$ plane.

The influence of the geometric dimensions of the nanowire on the electric potential and local electron density has also been investigated as shown in Figs. 3 and 4. While keeping the radius of the nanowire at $25 \mathrm{~nm}$, the donor concentration at $N_{\mathrm{D}}=10^{17} \mathrm{~cm}^{-3}$ and the applied force at $80 \mathrm{nN}$, the length of the nanowire has been varied in the range $200 \mathrm{~nm}<L<1000 \mathrm{~nm}$. Figures 3(a) and 3(b) show that the length of the nanowire does not influence either the electric potential distribution or the free electron density.

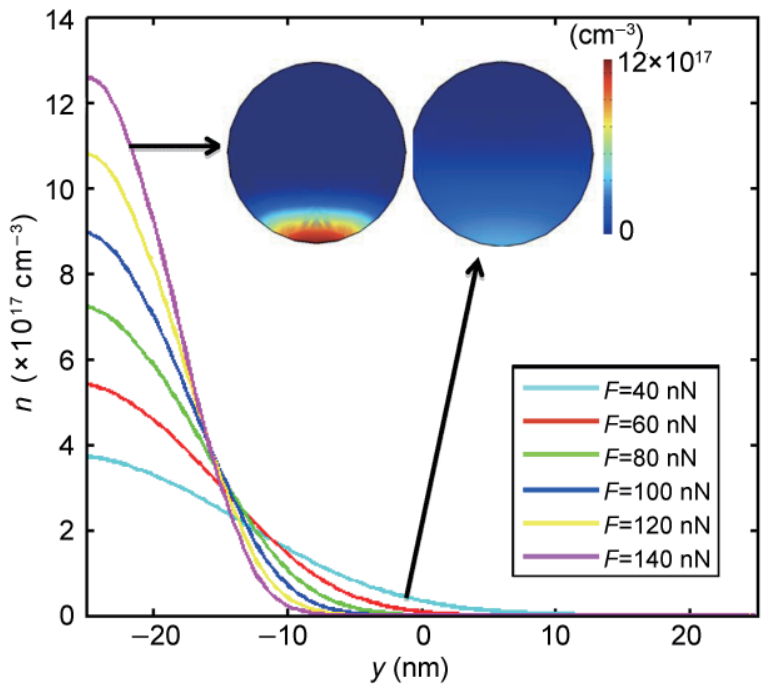

(b)

Figure 2 (a) Piezoelectric potential and (b) local electron density for different external forces $40 \mathrm{nN}<F<140 \mathrm{nN}$. (a) Also shows the color-plot of the calculated potential at a cross section of $x=0$ for $F=40 \mathrm{nN}$ and $F=140 \mathrm{nN}$. (b) Also shows the color-plot of the calculated free electron distribution at a cross section of the nanowire at a height of $400 \mathrm{~nm}$, for $F=40 \mathrm{nN}$ and $F=140 \mathrm{nN}$. Here only half of the space $x>0$ is calculated, taking advantage of the mirror symmetry of the $x=0$ plane. The plot in the $x<0$ region is derived by a simple reflection of the solution in the $x>0$ region

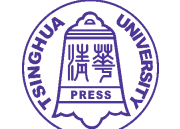




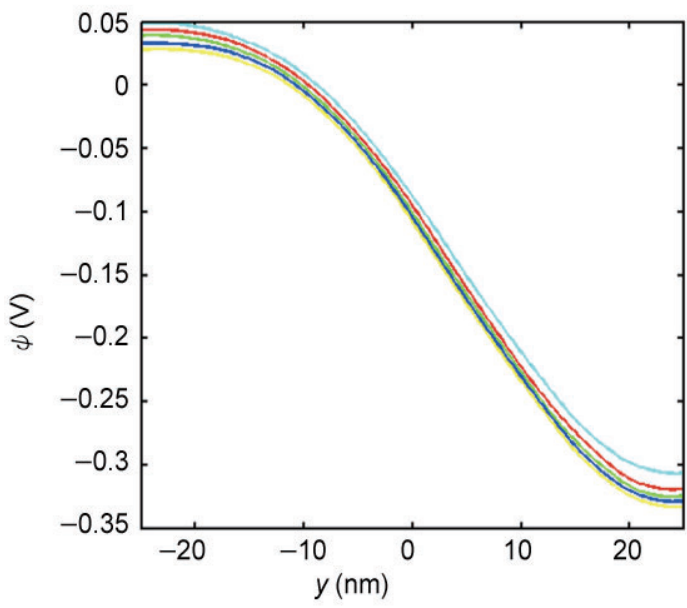

(a)

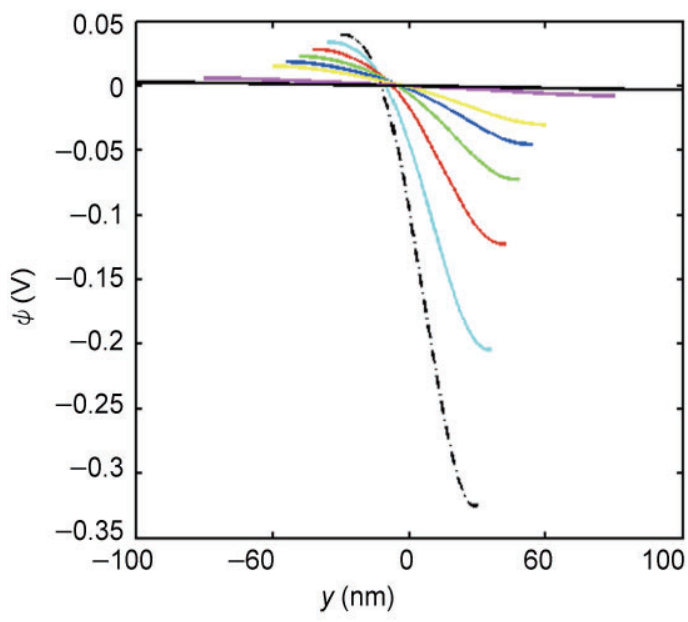

(c)

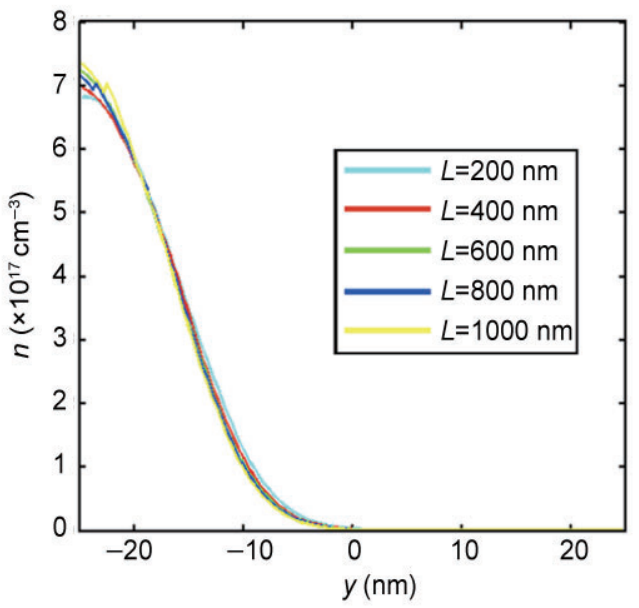

(b)

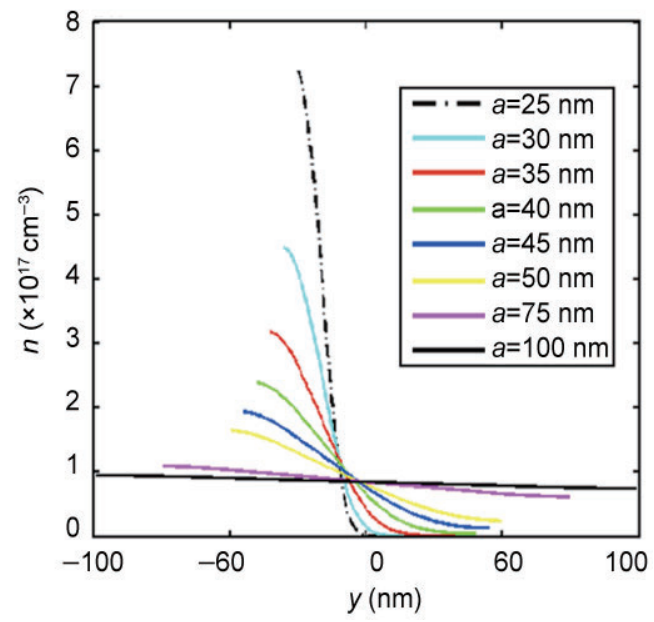

(d)

Figure 3 (a) Piezoelectric potential and (b) local electron density for different lengths of the nanowire $200 \mathrm{~nm}<L<1000 \mathrm{~nm}$; (c) piezoelectric potential and (d) local electron density for different radii of the nanowire $25 \mathrm{~nm}<a<100 \mathrm{~nm}$. The donor concentration is $N_{D}=10^{17} \mathrm{~cm}^{-3}$, at $T=300 \mathrm{~K}$; the external force is $f_{y}=80 \mathrm{nN}$. The line plot is along the diameter of the nanowire, at $z=400 \mathrm{~nm}$

When keeping the length of the nanowire constant at $600 \mathrm{~nm}$, the effect of varying the radius in the range $25 \mathrm{~nm}<a<100 \mathrm{~nm}$ has been investigated. The results are shown in Figs. 3(c) and 3(d) for electric potential and free electron concentration, respectively. An increase in the radius decreases both the parameters; the electric potential is almost neutralized for a radius of $100 \mathrm{~nm}$. It has to be noted that the increase of the radius also decreases the magnitude of the strain experienced by the nanowire, as we are keeping the applied force constant.

Figure 4 shows the influence of the aspect ratio $L / a$, on the electric potential and free electron distribution. It summarizes the results previously described in Fig. 3: the length variation does not affect the variables, while the increase in the radius decreases both of them.

In summary, the influence of different parameters on the equilibrium piezoelectric potential distribution in a deformed $\mathrm{ZnO}$ semiconductive NW has been systematically investigated. The donor concentration has been shown to markedly affect the electric potential distribution. In particular, a donor concentration $N_{\mathrm{D}}>10^{18} \mathrm{~cm}^{-3}$ will neutralize the electric potential across the nanowire. The increase in the applied force has been shown to increase the value of the electric potential in the compressed side, due to higher values of the strain. The influence of geometric parameters has also been investigated: the length of the nanowire does not affect the electric 


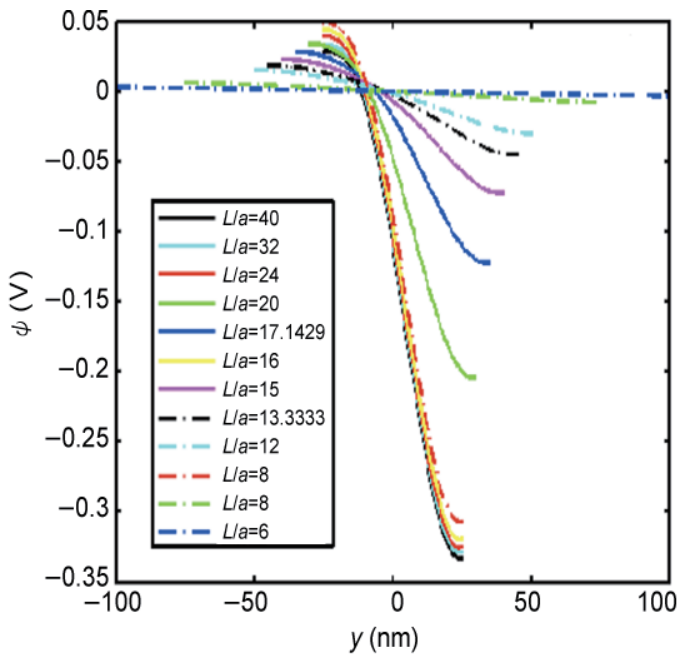

(a)

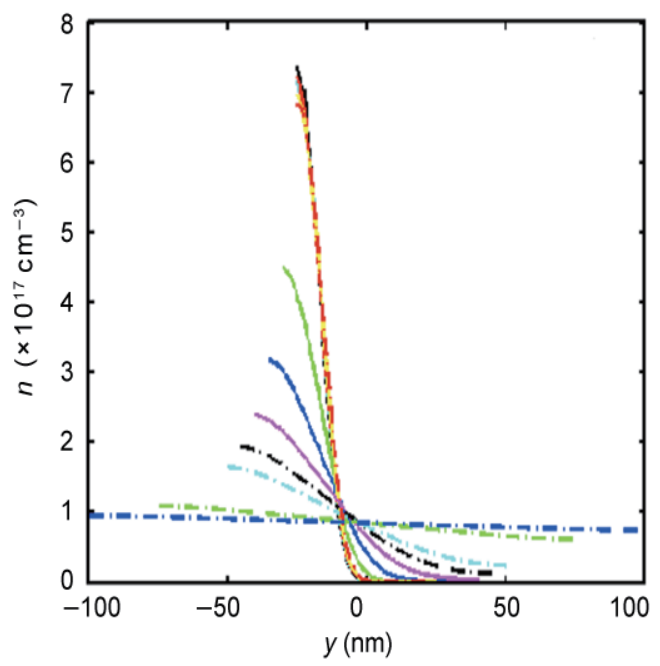

(b)

Figure 4 Piezoelectric potential and (b) local electron density for different aspect ratio of the nanowire $6<L / a<40$. The donor concentration is $N_{D}=10^{17} \mathrm{~cm}^{-3}$, at $T=300 \mathrm{~K}$; the external force is $f_{y}=80 \mathrm{nN}$. The line plot is along the diameter of the nanowire, at $z=400 \mathrm{~nm}$

potential distribution, while an increase in the radius of the nanowire decreases it. Finally, it must be pointed out that the above results were obtained for the equilibrium case. In reality, the dynamics have to be taken into account if the mechanical deformation process is rather fast.

\section{Acknowledgements}

The authors acknowledge the U.S. - Italy Fulbright Commission for awarding a Fulbright Scholarship to Giulia Mantini.

\section{References}

[1] Wang, Z. L. Self-powering nanotech. Sci. Am. 2008, 298, 82-87.

[2] Wang Z. L. Towards self-powered nanosystems: From nanogenerators to nanopiezotronics. Adv. Funct. Mater. 2008, 18, 3553-3567.

[3] Wang, Z. L.; Song, J. H. Piezoelectric nanogenerators based on zinc oxide nanowire arrays. Science 2006, 312, 242-246.

[4] Wang, X. D.; Song, J. H.; Liu, J.; Wang, Z. L. Directcurrent nanogenerator driven by ultrasonic waves. Science 2007, 316, 102-105.

[5] Qin, Y.; Wang, X. D.; Wang, Z. L. Microfibre-nanowire hybrid structure for energy scavenging. Nature 2008, 451, 809-813.

[6] Xu, S.; Wei, Y. G.; Liu, J.; Yang, R.; Wang, Z. L. Integrated multilayer nanogenerator fabricated using paired nanotipto-nanowire brushes. Nano Lett. 2008, 8, 4027-4032.

[7] Wang, Z. L. The new field of nanopiezotronics. Mater. Today 2007, 10, 20-28.

[8] Gao, Y.; Wang, Z. L. Electrostatic potential in a bent piezoelectric nanowire. The fundamental theory of nanogenerator and nanopiezotronics. Nano Lett. 2007, 7, 2499-2505.

[9] Falconi, C.; Mantini, G.; D'Amico A.; Wang, Z. L. Studying piezoelectric nanowires and nanowalls for energy harvesting. Sens. Actuators B: Chem. 2009, 139, 511-519.

[10] Landau, L. D.; Lifshitz, E. M.; Pitaevskii, L. P. Electrodynamics of Continuous Media, 2nd Ed.; Pergamon: New York, 1984.

[11] Van de Walle, C. G. Hydrogen as a cause of doping in zinc oxide. Phys. Rev. Lett. 2000, 85, 1012-1015.

[12] Cox, S. F. J.; Davis, E. A.; Cottrell, S. P.; King, P. J. C.; Lord, J. S.; Gil, J. M.; Alberto, H. V.; Vilão, R. C.; Duarte, J. P.; de Campos, N. A.; Weidinger, A.; Lichti, R.L.; Irvine, S. J. C. Experimental confirmation of the predicted shallow donor hydrogen state in zinc oxide. Phys. Rev. Lett. 2001, 86, 2601-2604.

[13] Look, D. C.; Farlow, G. C.; Reunchan, P.; Limpijumnong, S.; Zhang, S. B.; Nordlund, K. Evidence for native-defect donors in n-type ZnO. Phys. Rev. Lett. 2005, 95, 225502.

[14] Gao, Y.; Wang, Z. L. Equilibrium potential of free charge carriers in a bent piezoelectric semiconductive nanowire. Nano Lett. 2009, 9, 1103-1110.

[15] Lu, M. P.; Song, J.; Lu, M. Y.; Chen, M. T.; Gao, Y.; Chen, L. J.; Wang, Z. L. Piezoelectric nanogenerator using p-type ZnO nanowire arrays. Nano Lett. 2009, 9, 1223-1227. 